Recepción: 28 / 02 / 2018

Aceptación: 19 / 04 / 2018

Publicación: 05 / 06 / 2018
Ciencias de la computación

Artículo de Investigación

\title{
Uso de la informática durante el aprendizaje en el nivel de educación básica
}

\author{
Use of computers for learning at the level of basic education
}

Uso da informática durante o aprendizado no nível de ensino básico

\author{
Jhonny M. Quiñonez-Quintero I \\ jhonnymquiñonez@hotmail.com \\ David V. Romero-Jaén II \\ davidromero@hotmail.com \\ Jazmín A. Saltos-Gómez ${ }^{\text {III }}$ \\ ing.jasycar@gmail.com
}

\section{Correspondencia: jhonnymquiñonez@hotmail.com}

${ }^{\text {I }}$ Magister en Ciencias de La Educación, Licenciado en Ciencias de la Educación Especialidad Informática Educativa, Ingeniero de Sistemas y Computación, Tecnólogo en Computación, Docente de la Universidad Técnica Luís Vargas Torres de Esmeraldas, Esmeraldas, Ecuador.

II Ingeniero en Sistemas Informáticos, Tecnólogo en Computación, Docente de la Universidad Técnica Luís Vargas Torres de Esmeraldas, Esmeraldas, Ecuador.

III Ingeniera en Sistemas y Tecnologías de la Información, Docente de la Universidad Luis Vargas Torres de Esmeraldas, Esmeraldas, Ecuador. 


\section{Resumen}

La aparición de la computadora y la creación posterior del internet, han ampliado sustancialmente el acceso a la información, permitiendo la comunicación casi instantánea en todo el mundo y ha puesto el poder de las computadoras en las manos de muchas personas, específicamente durante el proceso de aprendizaje de los aprendices. Basado en esta perspectiva, el presente artículo se ubicó como un proyecto de investigación evaluativa y se catalogó como descriptiva de campo, debido a que sólo se observaron los componentes relacionados con los niveles del aprendizaje y la informática. El diseño de investigación fue no experimental. La población la constituyeron 58 alumnos del nivel de educación básica e instrumento aplicado fue un cuestionario estructurado por 10 ítems. Los resultados permitieron indicar un nivel de aprendizaje excelente por el 58,6\% de las opiniones promedios en sus tres características de asimilación, construcción y transferencia.

Palabras clave: informática; aprendizaje; nivel de asimilación; nivel de construcción y nivel de transferencia.

\section{Abstract}

The appearance of the computer and the subsequent creation of the internet, have substantially expanded access to information, enabling near-instant communication around the world and has been the power of computers in the hands of many people, specifically during the learning process of learners. Based on this perspective, this article is ranked as an evaluative research project and was catalogued as a descriptive field, since only the levels of learning and computerrelated components were observed. The research design was not experimental. 58 students of basic education level constituted the population and instrument applied was a questionnaire structured by 10 items. The results allowed to indicate a level of $58.6 \%$ of the views excellent learning averages in three characteristics of assimilation, build and transfer.

Keywords: information technology; learning; level of assimilation; level of construction and transfer level.

\section{Resumo}

A aparência do computador e a subsequente criação da Internet aumentaram substancialmente o acesso à informação, permitindo a comunicação quase instantânea em todo o mundo e colocando 
o poder dos computadores nas mãos de muitas pessoas, especificamente durante o processo de aprendizagem de aprendizes. Com base nessa perspectiva, este artigo foi classificado como um projeto de pesquisa avaliativa e classificado como campo descritivo, pois apenas os componentes relacionados aos níveis de aprendizagem e computação foram observados. O desenho da pesquisa foi não experimental. A população constituiu 58 alunos do nível de educação básica e instrumento aplicado foi um questionário estruturado por 10 itens. Os resultados permitiram indicar um excelente nível de aprendizado em $58,6 \%$ das opiniões médias em suas três características de assimilação, construção e transferência.

Palavras chave: informática; aprendizagem nível de assimilação; nível de construção e nível de transferência.

\section{Introducción}

La informática en la educación hace mención al uso o enlace de las tecnologías de la información en el proceso pedagógico de la enseñanza desde el proceso inicial del aprendizaje, es decir, desde la etapa de primaria. Para ello el docente debe ser facilitador y mediador de estos nuevos aprendizajes a los que los educandos se están enfrentando, pero para ello debe estar adecuadamente capacitado con respecto a estos nuevos avances en tecnología lo cual debe permitir que el aprendiz sea explorador y constructor de sus aprendizajes de acuerdo a los conocimientos previos que trae de su entorno social. La incorporación de las tecnologías como la informática en la escuela ha permitido hacer más significativa la búsqueda de información para las clases y en efecto muchas veces dentro de la misma aula gracias a la incorporación de estas mediante el uso guiado, libre o práctico, así como de otros medios audiovisuales acercando a los aprendices a la sociedad del conocimiento.

Cabe destacar, que los conocimientos en informática en general, buscan centrar la atención en aportar soluciones a incógnitas o situaciones problemas del ser humano en cuanto al uso, aplicación y asimilación de las tecnologías por intermedio del aprendizaje de los diferentes contenidos de la ciencia de la informática, que el entorno de una organización inteligente de interacción constante con el hombre, le permitirá mantener una constante capacidad de adaptación al conocimiento, así como conocer las diferentes técnicas y herramientas de la gestión del conocimiento, con miras a lograr fortalecer las relaciones entre los escenarios virtuales como 
zonas de aprendizajes versus las necesidades como el proceso de empoderamiento del conocimiento para su transformación y aplicación.

Ante lo expuesto, cabe destacar a González (2005), quien explica que el uso de las nuevas tecnologías, permite desarrollar en los niños las competencias y habilidades necesarias que les permitan desempeñarse en un mundo cada vez más tecnologizado y globalmente comunicado.

Tomando en cuenta esos puntos de vista señala que el uso del blog en la sala de clases pueden agregar un plus a la significación de los trabajos de los alumnos, porque en la medida en que se sientan parte de la comunidad global, en que se vean capaces de desarrollar por sí mismos un texto que los vincule al mundo desde su propia y personal opinión, los alumnos irán comprendiendo por qué hoy la información es tan importante y por qué discriminarla, ponderarla y procesarla hace la diferencia a la hora de adoptar posturas personales respecto de los temas. Con base a lo expuesto, en este artículo se caracterizó el uso de la informática durante el aprendizaje en el nivel de educación básica.

\section{Desarrollo}

La mente humana tiene capacidades limitadas para procesar y almacenar informaciones; sus posibilidades para el aprendizaje verbal significativo dependen de capacidades cognitivas, como pueden ser la representación simbólica, abstracción, categorización y generalización (Ausubel, 2002). En la adquisición de conocimientos, el aprendizaje es el mecanismo humano por excelencia, para construir, elaborar y asimilar conocimiento. El resultado del aprendizaje es el significado del concepto y no solamente la identificación de sus atributos.

Es preciso para desarrollar el aprendizaje de una manera efectiva en el estudiante, intensificar los procesos intrínsecos del saber, el cual busca entre otras maneras hacer, entender y compartir lo aprendido, es por ello vital conocer los niveles de aprendizajes que intervienen, entre estos se destacan lo de asimilación, construcción y transferencia.

Aprendizaje por asimilación. Según Ausubel (2002) para que se produzca un aprendizaje significativo, es preciso que tanto el material que debe aprenderse como el sujeto que debe aprenderlo cumplan ciertas condiciones. En cuanto al material, es preciso que posea significado en sí mismo, es decir, que sus elementos están organizados en una estructura. Pero no siempre los materiales estructurados con lógica se aprenden significativamente, para ello, es necesario 
además que se cumplan otras condiciones en la persona que debe aprenderlos. En primer lugar, es necesaria una predisposición para el aprendizaje, por lo que la persona debe tener algún motivo para aprender). Al respecto Díaz (1989), citado por Díaz y Hernández (2002), indica que los materiales de estudio y la información exterior se interrelacionan e interactúan con los esquemas de conocimientos previo y las características personales del aprendiz.

Aprendizaje por construcción. Según el modelo constructivista, indica Coll (2000) el aprendizaje es un proceso de construcción de conocimiento que tiene lugar cuando el alumno relaciona los nuevos contenidos con las experiencias y conocimientos que tiene almacenados previamente en la memoria. Piaget (1984) y Vygotsky (1979). Se pueden incluir como fuentes o precursores de lo que hoy llamamos psicología cognitiva y también pueden ser considerados como constructivistas en tanto son precursores de estas teorías contemporáneas. Ideas básicas de Jean Piaget. Su pensamiento es la base en que se asienta el aprendizaje y consiste en el conjunto de mecanismos que el organismo pone en movimiento para adaptarse al medio ambiente. El constructivismo es una respuesta a los problemas del ser humano ante la avalancha de información y medios electrónicos que facilitan y promueven la comunicación. Los antecedentes se encuentran en los trabajos de Vigotsky y de Piaget. Pone énfasis en la búsqueda epistemológica sobre cómo se conoce la realidad, como se aprende esto es, el origen y desarrollo del conocimiento y la cultura.

Aprendizaje por Transferencia. La aplicación de los conocimientos aprendidos para facilitar o dificultar otros aprendizajes posteriores es lo que se conoce como transferencia del aprendizaje. Si el aprendizaje previo facilita la adquisición de un nuevo aprendizaje, se dice que hay una transferencia positiva. Si, por el contrario, el aprendizaje previo dificulta la adquisición de un aprendizaje posterior, se dice que hay una transferencia negativa. La principal preocupación de Bruner (2001) es inducir al aprendiz a una participación activa en el proceso de aprendizaje, lo cual se evidencia en el énfasis que pone en el aprendizaje por descubrimiento. El aprendizaje se presenta en una situación ambiental que desafíe la inteligencia del aprendiz impulsándolo a resolver problemas y a lograr transferencia de lo aprendido. Se puede conocer el mundo de manera progresiva en tres etapas de maduración (desarrollo intelectual) por las cuales pasa el individuo, las cuales denomina el autor como modos psicológicos de conocer: modo nativo, 
modo icónico y modo simbólico, que se corresponden con las etapas del desarrollo en las cuales se pasa primero por la acción, luego por la imagen y finalmente por el lenguaje.

Estas etapas son acumulativas, de tal forma que cada etapa que es superada perdura toda la vida como forma de aprendizaje. Estos modos de conocer se relacionan estrechamente con los estadios del desarrollo de la teoría de Piaget: pre operacional, operaciones concretas y operaciones formales. Aunque dichos modos de conocer se adquieren progresivamente, igualmente una vez establecidos duran toda la vida. El modo nativo de conocer significa que la representación del mundo se realiza a través de la acción, de la respuesta motriz. El modo icónico se realiza a partir de la acción y mediante el desarrollo de imágenes que representan la secuencia de actos implicados en una determinada habilidad. La representación simbólica surge cuando se internaliza el lenguaje como instrumento de cognición.

\section{Metodología}

El presente artículo se ubicó dentro de lo que se denomina: proyecto de investigación evaluativa, ya que se orientó a caracterizar el uso de la informática durante el aprendizaje en las escuelas de educación básica adscritas al cantón Jipijapa, en la provincia de Manabí. Se catalogó como descriptiva de campo, debido a que sólo se observaron los componentes relacionados con los niveles del aprendizaje y la informática. El diseño de investigación utilizado se clasificó como no experimental, que, según Hernández, Fernández y Baptista, (2010) se analizaron en su estado natural. Se detalla como descriptivo transaccional por cuanto el estudio conllevó a caracterizar la situación actual en un solo momento. La población y los instrumentos del estudio estuvieron conformados por un cuestionario simple dirigido a 58 alumnos de educación básica del cantón Jipijapa, en la provincia de Manabí, compuesto por veinte (10) ítems de selección simple.

\section{Resultados}

Para el análisis de los datos obtenidos entre los estudiantes de educación básica del cantón de Jipijapa de la provincia de Manabí, se pudo constatar lo siguiente: 
Tabla 1. Nivel de aprendizaje

\begin{tabular}{|c|c|c|c|c|c|c|c|c|c|c|}
\hline ALTERNATIVAS & Exc & Iente & & deno & & ular & & alo & Mu & malo \\
\hline Niveles de: & $\mathbf{F r}$ & $\%$ & $\mathbf{F r}$ & $\%$ & Fr & $\%$ & Fr & $\%$ & $\mathbf{F r}$ & $\%$ \\
\hline Asimilación & 37 & 63.8 & 21 & 36.2 & 0 & 0 & 0 & 0 & 0 & 0 \\
\hline Construcción & 30 & 51,7 & 22 & 38 & 6 & 10.3 & 0 & 0 & 0 & 0 \\
\hline Transferencia & 35 & 60.3 & 13 & 22.4 & 10 & 17.3 & 0 & 0 & 0 & 0 \\
\hline Promedio & 34 & 58.6 & 19 & 32.7 & 5 & 8.7 & $\mathbf{0}$ & $\mathbf{0}$ & $\mathbf{0}$ & $\mathbf{0}$ \\
\hline MEDIA & \multicolumn{10}{|c|}{4,77} \\
\hline $\begin{array}{l}\text { DESVIACIÓN } \\
\text { ESTANDAR }\end{array}$ & \multicolumn{10}{|c|}{0.28} \\
\hline MEDIANA & \multicolumn{10}{|c|}{4,84} \\
\hline
\end{tabular}

Fuente: elaboración propia

Grafico 1. Nivel de aprendizaje

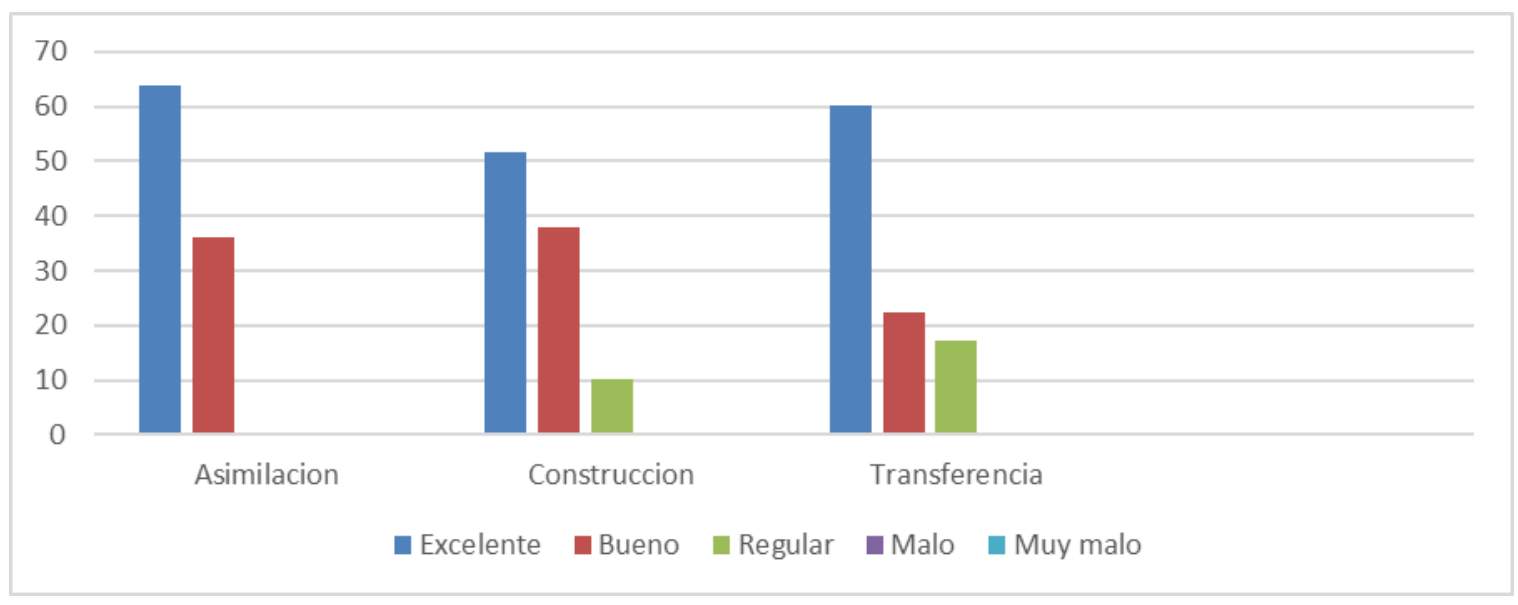

Fuente: elaboración propia

Se indica en la tabla y grafico 1, que el nivel de aprendizaje se evaluó con un promedio de 58,6\% de las opiniones para la alternativa excelente en sus tres características de asimilación, construcción y transferencia

Por otro lado, el $60.3 \%$ de los alumnos evaluados consideraron el nivel de transferencia como excelente para su aprendizaje por descubrimiento y de los contenidos que se han de aprender en clases, para percibirlos como un conjunto de soluciones a sus dudas de aprendizaje. Mientras que el $63.8 \%$ por igual considero el nivel de asimilación como excelente para su aprendizaje con el uso de la informática en las clases, por cuanto la información previa que poseen de los conceptos 
obtenidos en su formación académica, denotan mayor nivel de comprensión y efectividad a partir de su uso. Asimismo, el 51,7\% de los investigados consideran que es excelente el nivel de construcción del conocimiento desde la información y medios electrónicos en la facilitación y promoción de la comunicación para su aprendizaje, así como el uso y practicidad de los contenidos de informática más efectivos que apuntan a su desarrollo académico y personal durante su proceso de enseñanza aprendizaje; el restante $15 \%$ lo evaluó como bueno y únicamente el 5\% como regular.

La alternativa bueno, se presentó en menor frecuencia con el 36,2\%; 38\% y 22.4\% para los niveles de asimilación, construcción y transferencia respectivamente. SE destaca el hecho en la cual las alternativas malas y muy malas no presentaron opinión alguna, favoreciendo la evaluación de los niveles de informativa.

\section{Conclusiones}

El nivel de aprendizaje se evaluó como excelente en sus tres características de asimilación, construcción y transferencia, por parte de los alumnos investigados. Hecho corroborado, por la no evaluación entre las alternativas malo y muy malo que más desfavorecían la evaluación de lo investigado.

El nivel de transferencia, permite obtener un aprendizaje por descubrimiento al navegar acerca de los contenidos que se han de aprender en clases, proporcionando lucidez para aclarar las dudas que se presenten durante el proceso de enseñanza aprendizaje.

A partir del nivel de asimilación, es posible lograr entender los nuevos conocimientos impartidos de los contenidos programáticos considerando la información previa que poseen de los conceptos obtenidos a través de la navegación de los mismos, denotándoles mayor nivel de comprensión y efectividad por su uso.

Los investigados consideraron que el nivel de construcción del conocimiento considerando la información y medios electrónicos les facilitan en la facilitación y promueven la comunicación para su aprendizaje, así como el uso y practicidad de los contenidos de informática más efectivos que apuntan a su desarrollo académico y personal durante su proceso de enseñanza aprendizaje.

\section{Referencias Bibliográficas}

Ausubel P David (2002). Adquisición y Retención del Conocimiento. Una Perspectiva Cognitiva. Colección Educador. Editorial Ediciones Paidós 
Bruner, J. (2001). El Proceso Mental en el Aprendizaje. Editorial Narcea. Segunda Edición.

Coll, C., 2000, "Constructivismo e intervención educativa", El constructivismo en la práctica, España, Editorial Laboratorio educativo.

Díaz y Hernández (2002). Estrategias docentes para un aprendizaje significativo: una interpretación constructivista. Recuperado de: https://scholar.google.com.co/scholar?q=diaz $+\mathrm{y}+$ hernandez $+2002+$ estrategias + didacticas\&hl=en \&as_sdt $=0 \&$ as_vis $=1 \&$ oi $=$ scholart

FDB Arceo, GH Rojas... - ... McGrawHill. México, DF.

González, B. (2005) Actividad educativa con el blog. Recuperado en: http://pedablogia.wordpress.com/2005/08/16/actividad-educativa-con-el-blog [Consulta realizada el: 31-05-17]

Hernández, Fernández y Baptista, (2010). Metodología de la investigación. (5ªde). México DF, Mc Graw Hill.

Vygotsky, L.S. (1979). El desarrollo de los procesos psicológicos superiores. Barcelona: Crítica. 